\title{
Mobility-prediction and energy optimization for multi-channel multi-interface ad hoc networks in the presence of location errors
}

\author{
Hassan Faouzi ${ }^{1}$, Mohammed Boutalline ${ }^{2}$ \\ ${ }^{1}$ Sultan Moulay Slimane University, Morocco \\ ${ }^{2}$ National Schools of Applied Sciences of Beni Mellal, Sultan Moulay Slimane University, Morocco
}

\begin{tabular}{|c|c|}
\hline Article Info & ABSTRACT \\
\hline Article history: & \multirow{11}{*}{$\begin{array}{l}\text { We present a mobility-prediction and energy optimization solution for multi-channel } \\
\text { multi-interface (MCMI) ad hoc networks in the presence of location errors. This solu- } \\
\text { tion includes routing of the MCMI communication links that adapt to dynamic chan- } \\
\text { nel, traffic conditions, interference and mobility of nodes. We start first with imple- } \\
\text { menting a novel cross-layer routing solution in order to share information between } \\
\text { network and MAC layer, the benefit of this technique is to collect information about } \\
\text { the channel quality and residual energy of the nodes and send them directly to the } \\
\text { network layer. Next, we present a mobility-prediction model using Kalman filter to } \\
\text { predict accurate locations and enhance routing performance, through estimating link } \\
\text { duration and selecting reliable routes. The performance of proposed mechanism is } \\
\text { measured using NS2.35 simulations with different scenarios and varying load in a net- } \\
\text { work. Comparative analysis of simulation results shows better performance of our } \\
\text { protocol (ME-MCMI AODV) in terms of reducing end-to-end delay, total dropped } \\
\text { packets and increasing network lifetime and packet delivery ratio (PDR). }\end{array}$} \\
\hline Received Oct 11, 2020 & \\
\hline Revised Jan 27, 2021 & \\
\hline Accepted Mar 2, 2021 & \\
\hline Keywords: & \\
\hline AODV & \\
\hline End to end delay & \\
\hline Energy consumption & \\
\hline Kalman filter & \\
\hline Mobile ad-hoc & \\
\hline NS2 (Simulator) & \\
\hline
\end{tabular}

This is an open access article under the CC BY-SA license.

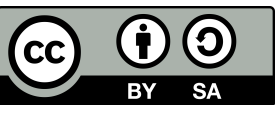

Corresponding Author:

Hassan Faouzi

Sultan Moulay Slimane University

Beni Mellal, Morocco

Email: faouzi.hassan.mi@gmail.com

\section{INTRODUCTION}

Wireless network works under two modes, infrastructure and the other without the aid of centralized administration. Mobile ad hoc networks (MANET) does not have a fixed topology and include a set of wireless mobile nodes which transfer data dynamically among themselves. Unlike VANET networks where we can control traffic by reducing the speed of movement of vehicles either by the driver or by utilizing mechanisms like the ones described in the papers $[1,2]$ which the authors proposed a new approach to estimate, track, and control users moving above speed limits in LTE-Advanced (LTE-A) networks. To reach these objectives they use mapping of the uplink CQI index of the UE since the CQI range can provide an indication to the system regarding the movement and the speed of the UE. However, MANET networks do not have this specificity because nodes can move freely in space.

The main objective of mobile ad hoc networks (MANET) is to extend the concepts of mobility to enable access to information and communication "anywhere and anytime" using routing protocols as ad-hoc on-demand distance vector (AODV) [3], dynamic source routing (DSR) [4], destination sequenced distance- 
vector (DSDV) [5], and optimized link state routing (OLSR) [6] and temporally ordered routing algorithm (TORA) [7] to transfer data. So, it is important to increase network lifetime by minimizing the control packets, because the mobile nodes are powered by independent power sources such as batteries or other consumable ones. In addition, since nodes have limited battery power and move frequently in the network, the links between them are frequently broken and causes a loss of data packets. Therefore, the node should send only necessary packets and select the path with lower link failure based on the localization of neighboring nodes to save its energy resource. However, dynamic topology of MANET makes it harder to determine the location of nodes in real time. Hence, our proposed method uses a mobility prediction to measure the link duration time, which can reduce overhead messages and improve routing performance.

Most of the existing researches mainly concentrate on the influence of node mobility on link reliability, they have ignored the residual energy of nodes in their methods and instead of working on multi-channel multiinterface, they adapt their mechanisms to use single interface single channel (SISC) environment. In this paper, energy and mobility are introduced as a new routing metric to select links in terms of reliability in a multichannel multi-interface ad hoc networks. Firstly, the node in our approach uses mobility to predict the link duration time. Then, it combines this duration with the residual energy to find a stable route that has a long lifetime. Finally, it searches a good mapping between channels and interfaces to send data to destination nodes.

The remainder of this paper is organized as follows: Section 2 introduces the related work and discuss some details about Mobility-prediction and energy optimization in MANET. Section 3 describes functional details of the proposed ME-MCMI AODV to improve the AODV protocol. Section 4 presents the experimental modeling and results of our proposed protocol using network simulator NS2. Finally, conclusions and some plans for future development in this field are given in Section 5.

\section{RELATED WORK}

In literature, a lot of research has been done to improve the performance of mobile Ad hoc networks. These searches seek to find routes satisfying certain constraints. Some have used probabilistic approaches to limit the number of routing packets while others have used queue length, bandwidth, mobility, energy and hop count that separates the source and the destination. Rare researchers who have taken into account the location errors in their models. In [8], the authors implemented a mobility-assisted using AODV protocol and taking into consideration location errors. To that end, they implement Kalman filter to predict accurate locations and take for granted level confidence in discovering routes to choose the best route.

The authors in [9] proposed two extensions of AODV protocol [3] to find routes based on residual energy and hop-count, the first uses Floyd Warshall and the other Bellman-Ford algorithm. These protocols implemented in the network in which, each node equipped with a multiple network interface [10] to overcome the problems of SISC. Their idea is to add a novel cross-layer routing solution which allows communication between physical and network layers. The authors of [11] have developed two topologies namely chain and grid, in which they further worked on directional and Omni-directional antenna. After simulation, the results show that directional antenna is more efficient in enhancing the spatial diversity and reducing collisions. In an another work [12], the authors developed two extension of AODV protocol, the first is AODVEA protocol, which incorporates local forwarding decision based on max min energy of nodes in order to increase the lifetime of the network. The second (AODVM) combines the same local forwarding decision parameters used in AODVEA protocol and shortest distance. Instead of using hop count as a parameter to calculate the best routes from source to destination, the authors of [13-15] utilize the energy and power level of the nodes. Simulations have shown that these improvements give better results compared to other algorithms in the same field of research. In [16] received signal strength from the (MAC) layer is used to estimate the stability of the radio connection. Their objective is to select the stable route to avoid paths which have a higher probability to be broken. The authors propose another solution to improve Quality Of Service by incorporating residual energies of source and destination nodes to calculate available link bandwidth.

A recent study [17] proposed a method to frame up a stable link network using a temporal data analysis model. In this model, the authors analyzed the mobility, position of neighbor nodes and used the statistical model auto regressive moving average (ARMA) to predict the stable neighbors of each node in a future time frame. They applied a Biogeographic-based optimization (BBO) technique to estimate relevant parameters in the ideal path from source to destination nodes. According to them, this optimal link offers a stable and reliable connection for the remaining lifetime of the data transfer in the network. 
A dynamic power ad hoc on-demand distance vector (DP-AODV) protocol which is an improvement of the existing AODV routing protocol was applied in [18]. In this extension, the authors modified the packets headers in the routing layer to include the distance information of the destination and neighbors count. They also modified hello packets of AODV to carry the "x-y position" coordinate field information in order to obtain the exact location of a node, which used by the routing protocol to determine the route. At the wireless physical layer, the algorithm selects the power required to keep the connectivity among the nodes and consequently reduce the overall power. The authors of [19] use a routing conditioned upon the achievement of many requirements. Among these, is the intermediate UAV able to respond with a throughput requested by the source UAV? Does the speed not exceed a predefined threshold? Simulation results show that their protocol (MDRMA) gives better results in terms of speed of establishment and stability of routes.

In [20], the authors proposed improvements of ad hoc on demand distance vector. These improvements take into account a metric based on energy consumption during route discovery in order to decrease load of control packets and increase both the network lifetime and packet delivery ratio. They did the simulation in an environment close to reality by using the Gilbert-Elliot model. To reduce the route-establishment overhead in AODV, the authors of $[21,22]$ attempt to minimize the number of intermediate nodes that participate in the route discovery process. This is achieved by reducing the number of route request (RREQ) depending on the length queue and energy of nodes or count of RREQ (nodes stop transfer the requests if the count of RREQ exceeds a threshold). Additionally, [23] presented two techniques for computing the link availability and decreasing the broadcast of RREQ packets. In the first technique, the link availability ratio (LAR) for all neighboring links is calculated using the present position of the neighbor and its angular sector in the transmission range. In the second technique, the transmission range of each node is divided into the outer, inner and middle zone. So, based on the received signal strength and two predefined thresholds only the nodes in the middle zone participate in the route discovery process.

The Multi-path routing allows data to be sent over a set of paths leading from source to destination. This is why other authors choose to work on this component [24-26] so as to limit the problem of road disruption and distribute the traffic between source as well as destination. In summary, the techniques used to decrease the dropped packets, end-to-end delay and increase the network lifetime and packet delivery ratio (PDR) in ad hoc networks focused only on one performance parameter or based on a single-interface single-channel environment, and in order to build a better routing protocol, we must satisfy all quality services. This is what we tried to implement in this paper.

\section{PROPOSED APPROACH AND FUNCTIONAL DETAILS}

In this section, we present our model to improve AODV protocol by using link duration time and residual energy as a metric of routing in multi-channel multi-interface communications in mobile Ad Hoc networks.

\subsection{Cross-layer}

To take benefit of information about the channel quality and residual energy of the nodes, we developed a cross-layer to share this information between network, MAC and physic layer. Although several methods using single channel single interface schemes tried to achieve a high quality of service scheme, most of them, if not all, were not successful due to intra-flow interference and inter-flow interference. So, in our work we used the multichannel environment to solve these problems and providing a more reliable MAC protocol for the users.

In MANET, channels are separated in frequency, so to use the different channels offered by the ad hoc network we need to develop a channel assignment approaches which allow coordination between nodes [27]. These approaches classified into three categories: Static, dynamic and hybrid channel assignment. In this work, we focus on hybrid channel strategy to benefit from the advantages of static and dynamic assignment. In this strategy, each node has a multiple interface, only one is designed to be fixed and the others become switchable. When a source node needs to communicate with a destination, it will switch its switchable interface to the same channel as pointed by fixed interface of the target node.

Figure 1 illustrates an example of communication between nodes when using "fixed" and "switchable interfaces". Assuming that node $\mathrm{X}$ has a data to be sent to node $\mathrm{Y}$. The fixed interfaces of nodes $\mathrm{X}$ and $\mathrm{Y}$ are assigning to channels 3 and 1 respectively. To ensure this communication, the switchable interface of node $\mathrm{X}$ is assigning to channel 1 , before transmitting the packet, because channel 1 is the fixed channel of node $\mathrm{Y}$. 
So, node Y can receive the packet since its fixed interface is listening to channel 1 . In the replay step, node $\mathrm{Y}$ switches its switchable interface to channel 3 and send a replay request, which is received by node $\mathrm{X}$ using its fixed interface on channel 3.

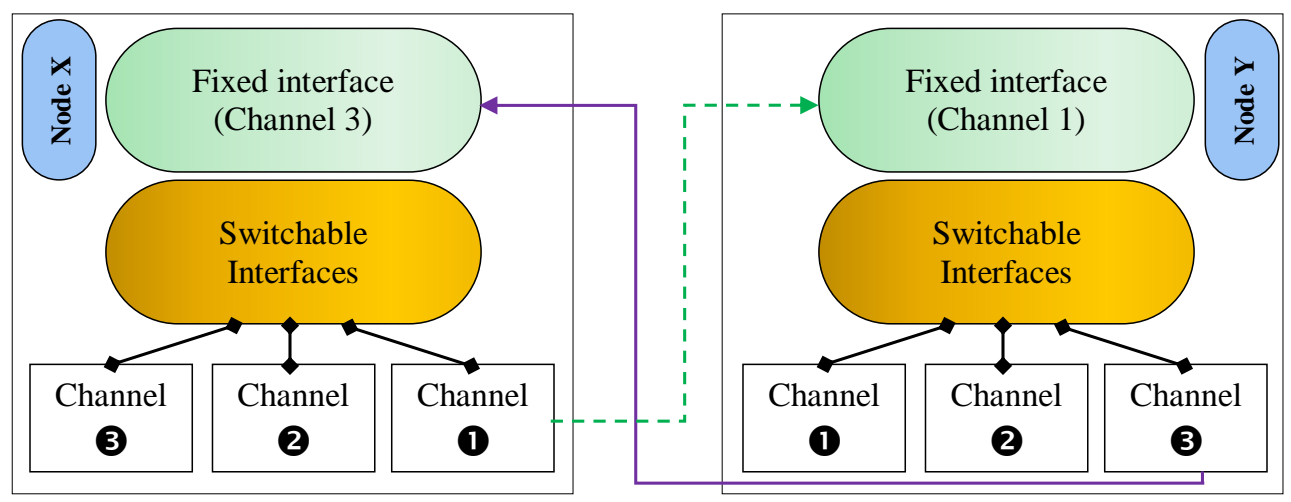

Figure 1. Communication between two nodes using fixed and switchable interfaces

\subsection{Residual energy}

The nodes consume the energy during transmission and reception activities. Therefore, energy is one of the actual considerable constrained in MANET. When a node participates in route establishment in many times, it may run off its power in later stages resulting in the breakdown of the link. So, our approach is energy aware reactive protocol which considers the nodes residual energy to select path to the destination, by applying this method, nodes can select paths with maximum lifetime, thus achieving considerable energy savings.

To attain this objective, we modified the route discovery process to select the path that consists of nodes with higher remaining energy. In this method, when a RREQ message is transmitted in the network, not every node, which receives the message, will diffuse it. If the residual energy of intermediate node is lower than a predefined threshold value, the RREQ is dropped, otherwise, the message is forwarded Figure 2.

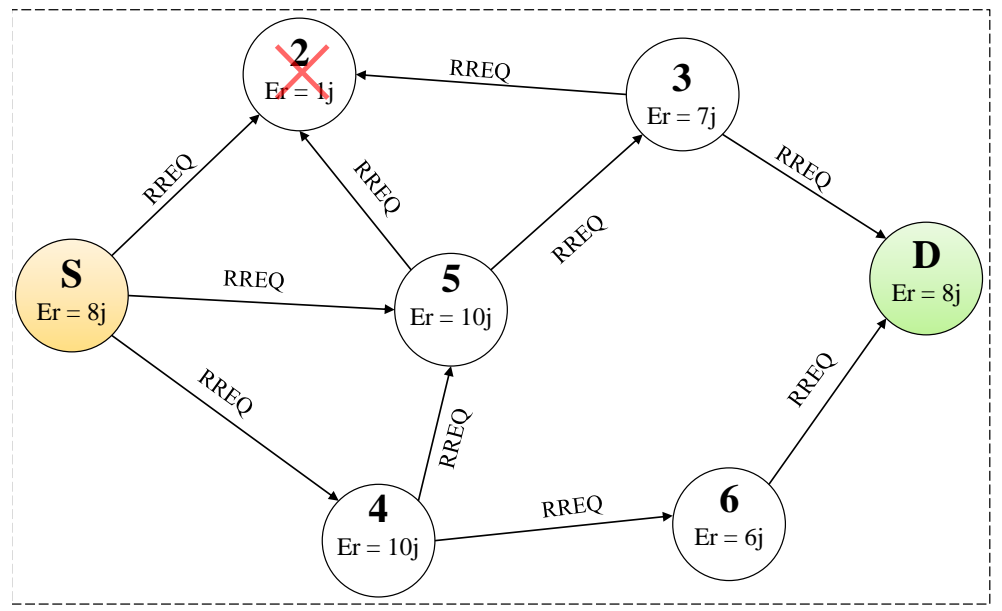

Figure 2. Route discovery process in ME-MCMI AODV

\subsection{Location correction}

In reality, the location measurement tools do not give the exact location of the nodes. So, to give credibility to our approach, we have introduced a model that takes into account the presence of location errors. The measurement error (also called Observational Error) is the difference between a measured quantity and its true value. 
In our model, we used the Kalman filter to estimate the internal state location of nodes in the network. Each node runs a Kalman filter predictor, which is employed to predict the node's own position (x, y) and velocity (vx, vy). The position vector at moment $t$ is measured by:

$$
X_{t}=[x(t), y(t), v x(t), v y(t)]^{T}
$$

To find the best estimate of the current state in regular intervals, we apply the time and measurement update mechanisms of Kalman filter as shown in (2) and (7). The steps involved in state estimation of our system are described as follows:

- Time update (Prediction)

$$
\text { Location Prediction : } X_{t}^{\prime}=A X_{t-1}+W_{t}
$$

$$
\text { Error Covariance : } P_{t}^{\prime}=A P_{t-1} A^{T}+Q_{t}
$$

- Measurement update (Correct)

$$
\begin{gathered}
\text { Measurement of state : } Y_{t}=C X_{t}^{\prime} \\
\text { Kalman Gain : } K=P_{k}^{\prime} H /\left(H P_{t}^{\prime} H^{T}+R\right) \\
\text { Update Prediction Measurement : } X_{t}=X_{t}^{\prime}+K\left(Y_{t}-H X_{t}^{\prime}\right) \\
\text { Update Error Covariance : } P_{t}=(I-K H) P_{t}^{\prime}
\end{gathered}
$$

In above equations:

$A$ : the state transition matrix;

$Q$ : the noise covariance matrix;

$H$ : the observation matrix;

$R$ : the noise covariance matrix of the observation.

$Y_{t}$ : the observation vector achieved from the current node, namely, the node's current position.

The figure as shown in Figure 3 the general flow and overview of our system model.

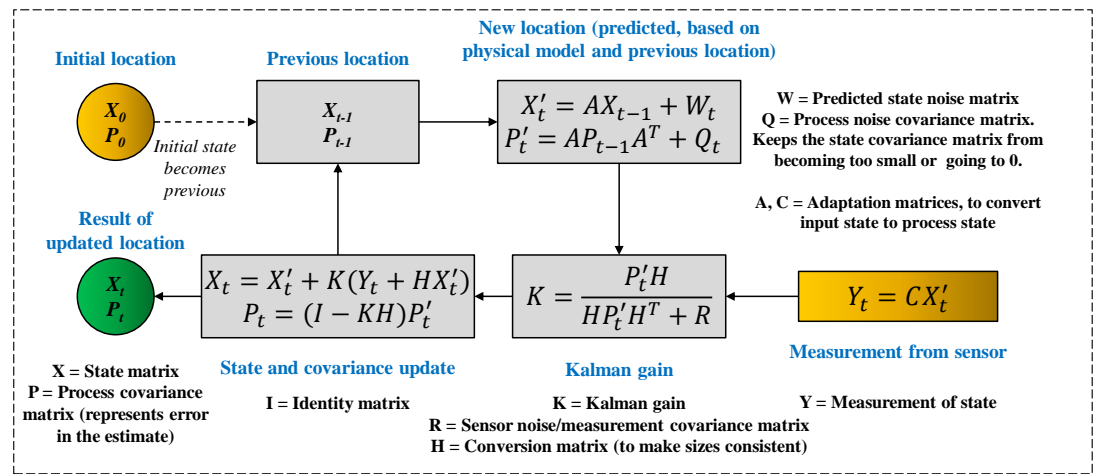

Figure 3. Overview of our system model 


\subsection{Predicting link expiration time}

We shall ensure that the selected route will be the stable one by calculating its link expiration time. Only if this time is greater than the predefined threshold value, then that node will take part in route discovery. The link expiration time is calculated at each hop of the route and taking into consideration the mobility of the nodes involved in a network. So, the node computes the link duration time, after reception the RREQ, between itself and the sender which implies the predicted lifetime of the link using the real location calculated in the previous section as shown in (6) instead of the measured location information. Let us assume two nodes $i$ and $\mathrm{j}$ are within the transmission range $\mathrm{r}$ of each other. Let:

- $\left(x_{i}, y_{i}\right)$ : The coordinate of node $i$.

- $\left(x_{j}, y_{j}\right)$ : The coordinate of node $j$.

$-V_{i}$ and $V_{j}$ be the speeds of nodes $i$ and $j$ respectively.

$-\theta_{i}$ and $\theta_{j}\left(0<=\theta_{i}, \theta_{j}<2 \pi\right)$ be the moving directions of nodes $i$ and $j$ respectively.

So, the link expiration time (length of the longest time interval during which the two nodes are within the transmission range of each other) $D_{t}$, of the link between the two nodes, as defined in [28], is given as shown in (8):

$$
D_{t}=\frac{-(a b+c d)+\sqrt{\left(a^{2}+c^{2}\right) r^{2}-(a d-b c)^{2}}}{a^{2}+c^{2}}
$$

$$
\text { Where : } \quad \begin{aligned}
a & =v_{i} \cos \theta_{i}-v_{j} \cos \theta_{j} \\
b & =X_{i}-X_{j} \\
c & =v_{i} \sin \theta_{i}-v_{j} \sin \theta_{j} \\
d & =Y_{i}-Y_{j}
\end{aligned}
$$

If the Link Expiration Time value is smaller than the Link Duration Time in the modified RREQ, the receiving node replaces the Link Duration Time value by the new one. In case the receiver is not the destination of the RREQ, the node broadcasts it to other nodes.

\section{EXPERIMENTAL MODELING, ANALYSIS AND RESULTS}

\subsection{Experimental modeling}

\subsubsection{Simulation model}

In this paper, the simulation of our proposed protocol (ME-MCMI AODV), AODV and AODV [8] is done by using network simulator (NS-2) software due to its availability. NS-2 is a discrete event Simulator written in $\mathrm{C}++$ and OTCL, $\mathrm{C}++$ for data per event packets and OTCL are used for periodic and triggered event. NS-2 includes a network animator called Nam Animator, which provides visual view of simulation. AWK scripts are used to analyze output of TCL and get the network performance.

\subsubsection{Model parameters}

In these simulations, we used a wireless network, which is a $1 \mathrm{~km} \times 1 \mathrm{~km}$ simulation environment. We employed MAC 802.11 protocol, with node transmission range of $250 \mathrm{~m}$. The constant bit rate (CBR) traffic under the user datagram protocol (UDP) is used to accurately compare different routing protocols with a sending rate of 4 packets per second, 512 bytes of packet size and simulation time of 600s. The random waypoint mobility (RWP) [29] model is used as a mobility model with randomly selected speed between $1 \mathrm{~m} / \mathrm{s}$ and 20 $\mathrm{m} / \mathrm{s}$. The performances of protocols are evaluated by varying both the network size (number of nodes) and the pause time. We consider 10 random simulation runs and the performance of the considered factor is the average of these outputs. The parameter settings are listed in Table 1. 
Table 1. The parameter settings of our simulation

\begin{tabular}{lc}
\hline Parameter & Values \\
\hline Network simulator & NS-2.35 \\
Simulation area & $1 \mathrm{~km} \mathrm{x} 1 \mathrm{~km}$ \\
Number of mobile nodes & $10,20,30,40,50,100,150$ \\
Simulation time (s) & 600 \\
Mobility model & Random way point \\
Pause time (s) & $10,20,30,40,50,100,150$ \\
Packet generation rate & 4 packets/s \\
Packet size (bytes) & $700,800,900,1000,1100,1200$ \\
Transmission range (m) & 250 \\
Number of interfaces & $2,3,4$ \\
\hline
\end{tabular}

\subsection{Results and discussion}

\subsection{Choosing the number of interfaces}

In our model, devices can communicate by means of multiple interfaces. So, we have to choice the optimal number of interfaces to implement in physical layer. The Table 2 shows the results of our simulation study in multi-channel multi-interface environment. From the table we can notice that beyond two interfaces the performance of our protocol decreases. This is due to several factors such as RF interference, antenna correlation. In addition, each antenna in the MIMO system needs a radio-frequency (RF) unit, so the battery gets drain faster due to processing of complex and computationally intensive signal processing algorithms. That's why we opted to use only two interfaces per node in the rest of our contribution.

Table 2. Performance analysis vs number of interfaces

\begin{tabular}{ccccc}
\hline Number of interface & PDR \% & End to end delay & Total Dropped Packets & Lifetime \\
\hline 2 & 96.9411 & 338.87 & 364 & 105.949859 \\
3 & 94.19 & 345.66 & 387 & 109.243 \\
4 & 93.45 & 376.634 & 392 & 111.324 \\
\hline
\end{tabular}

\subsubsection{Packet delivery ratio}

Packet delivery ratio (PDR) is calculated by dividing the number of packets received by the CBR sink at the final destination by the number of packets originated by the "application layer" CBR sources. The PDR needs to be high for effective performance of routing. Figure 4 shows delivery ratio of the data packets of ME-MCMI AODV, AODV and AODV [3] in terms of variation of number of nodes, pause time and packet size. We observed that the protocols have higher PDR when the nodes move at low speeds. When the speed increases, routing protocols suffer a decrease in PDR. This is normal because higher speeds of nodes make routes unstable which leads to an increase of loss data.

We notice also that our proposed protocol shows higher values regardless of variation of parameters (node density, pause time and packet size) as compared to AODV and AODV [8], because proposed protocol select the nodes which have sufficient energy and high link duration time. This mechanism reduces the chances of route failure especially in case of high mobility (low pause time), which result in improving the packet delivery efficiency.

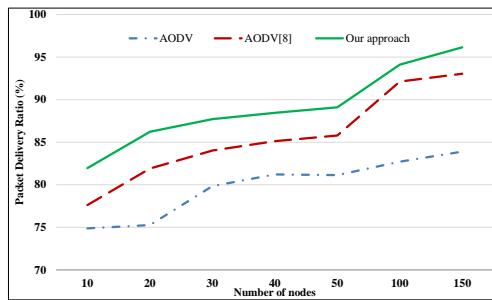

(a)

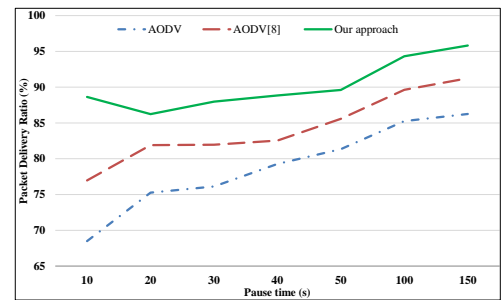

(b)

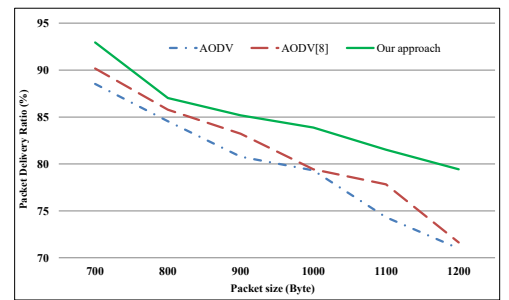

(c)

Figure 4. Packet Delivery Ratio by changing the: (a) number of nodes, (b) pause time and (c) packet size 


\subsubsection{Total dropped packets}

The Total Dropped Packets is the number of packets that is not received by the destination. The packets may be lost due to many factors such as transmission errors and congestion. This loss may take place at both, network and MAC layer. The result of these factors is related with the host mobility, number of connections, traffic load and packet size. From Figure 5, the variation in number of mobile nodes, pause time and packet size depicts that the AODV and AODV [8] have more dropped packets than ME-MCMI AODV. The number of nodes in the network, pause time and packet size will affect the requirement of route discovery between different pairs in the network. So, it can be seen from this figure that the number of dropped packets increases when the pause time decreases and packet size increases, because higher mobility leads to more broken links and higher packet size makes the chances of loss very significant due to collisions and interface overflows. In our approach, the paths of the high residual energy and link duration time are selected, so the route will not broke quickly, thus it reduces the number of dropped packets.

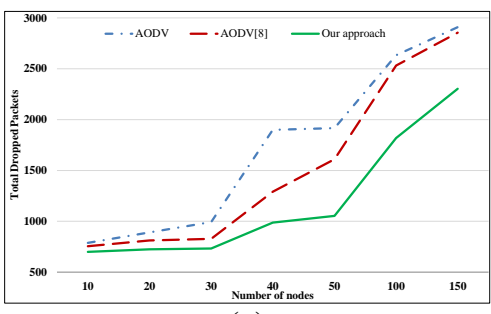

(a)

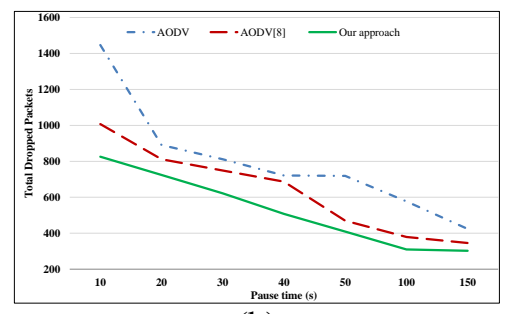

(b)

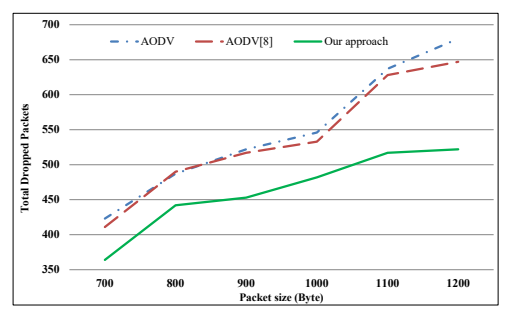

(c)

Figure 5. Total Dropped Packets by changing the: (a) number of nodes, (b) pause time and (c) packet size

\subsubsection{End to end delay}

The end to end delay is the ratio of time difference between numbers of packet send and received over the total time require to reach the destination. It is a significant parameter for evaluating a protocol, the more delay is reduced, the performance of network gives better output. Figure 6 shows end to end delay for number of nodes from 10 to 150 , pause time from 10 s to 150 s and packet size from 700 to 1200 bytes. From this figure, we notice that this parameter is decreased as the packet size, density of the network and mobility of nodes increased. Firstly because the probability of success in accessing the medium decreased when a greater number of nodes contend for access to the channel in stable network, but also because a larger packet needs more time to reach destination than smaller packets due to more packet drops and packet retransmissions are needed. We observe also that the average end to end delay of ME-MCMI AODV is smaller than both AODV and AODV [8] in all simulation scenarios (number of nodes, pause time and packet size). The reason is that our protocol reduce the traffic load by selecting the stable paths and this reduces queuing and propagation delays.

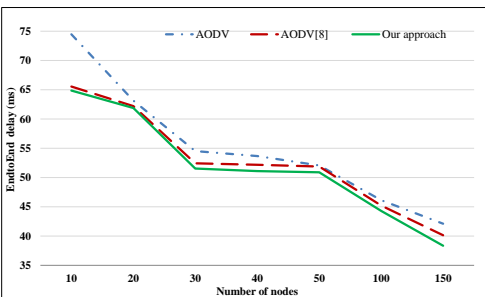

(a)

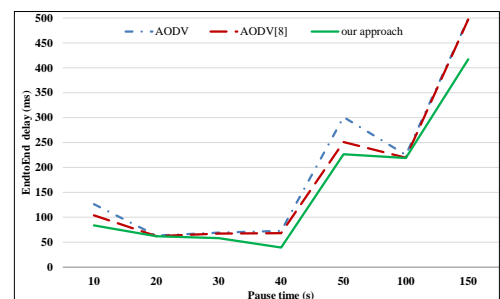

(b)

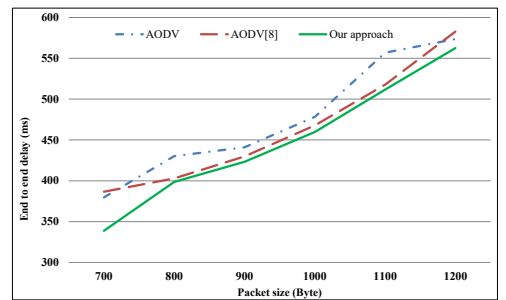

(c)

Figure 6. End to end delay by changing the: (a) number of nodes, (b) pause time and (c) packet size

\subsubsection{Lifetime}

From Figure 7, the results show that the network lifetime increases as the node density or pause time increases. Because if the number of nodes in the network is too small, feasible routes between sources and destinations may not exist in this network, so larger overhead messages need to keep and discover routes, which lead to high consumption of energy. For the third parameter, we observe that as packets size increases, 
more energy will be required to transmit data packets from one end to the other, hence reducing the lifetime of the network nodes and the overall lifetime of the network. The results reveal that our modified algorithm outperforms both AODV and AODV [8] by achieving long duration of time for the first node witch exhausts its energy on the network. The improvement in network lifetime is due to the fact that ME-MCMI AODV prevents small residual energy nodes to be a relay node or selects a path that has long duration time than shorter path between source and destination.

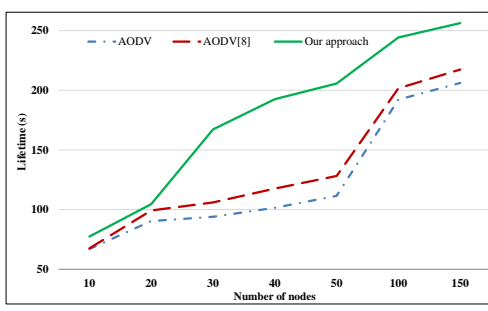

(a)

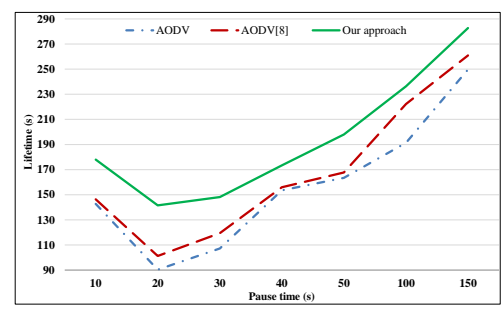

(b)

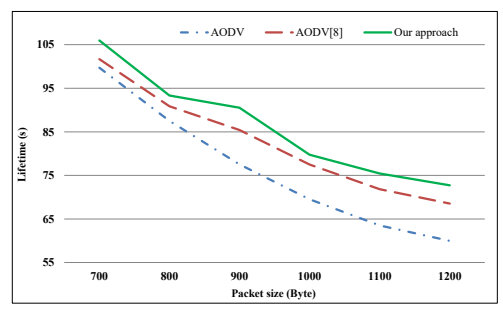

(c)

Figure 7. Lifetime by changing the: (a) number of nodes, (b) pause time and (c) packet size

\subsubsection{Reason behind the result}

As mentioned before, the results demonstrate that the ME-MCMI AODV generates better performance results as compared with a basic AODV and AODV [8]. The improved performance of ME-MCMI AODV compared to the two others protocols can be attributed to several design factors. One of the major factors is the incorporation of the enhanced RREQ mechanism, which lowers the rate of problems in our approach. In ME-MCMI AODV, the route created between any pair of nodes consists only of nodes whose energy level is higher than the threshold, so our protocol ensures a more stable link, without unnecessary link breakages, and as a result more successful packet delivery to destination nodes. Consequently, ME-MCMI AODV sends out less number of control packets that can reduce the overhead and increase lifetime of the network.

\section{CONCLUSION}

The stability of the route and lifetime of the network are considered as challenging tasks in MANET. This paper proposed a multi-channel multi-interface on-demand routing algorithm (ME-MCMI AODV) with a mobility prediction that takes into account the location errors and residual energy of nodes. Via simulations, our proposed algorithm shows significant performance improvements in terms of packet delivery ratio, total dropped packets, end-to-end delay and network lifetime compared with other protocols in the field, especially in a network with more connections, high mobility of nodes and large packet size. Because ME-MCMI AODV protocol select the nodes which have sufficient energy and high link duration time so the route will not broke quickly. Taking for granted the benefit of the solution proposed in this paper, in the future work we will try to expand the solution by proposing a model that will take into account other parameters in the process of establishing routes between source and destination nodes such as channel queue length, signal to noise ratio and varying parameters of simulations (adding some results versus the maximum speed in mobility model, generation and rate.

\section{REFERENCES}

[1] A. O. al Janaby, A. Al-Omary, S. Y. Ameen, and H. M. Al-Rizzo, "Tracking high-speed users using snr-cqi mapping in lte-a networks," 2018 International Conference on Innovation and Intelligence for Informatics, Computing, and Technologies (3ICT), 2018, pp. 1-7, doi: 10.1109/3ICT.2018.8855771.

[2] A. O. Al Janaby, A. Al-Omary, S. Y. Ameen, and H. Al-Rizzo, "Tracking and controlling high-speed vehicles via cqi in lte-a systems," International Journal of Computing and Digital Systems, vol. 9, pp. 1-10, Jul. 2020.

[3] C. E. Perkins, E. M. Royer, "Ad hoc ondemand distance vector (aodv) routing," Proceedings WMCSA'99. Second IEEE Workshop on Mobile Computing Systems and Applications, vol. 3561, 2003, doi: 10.1109/MCSA.1999.749281.

[4] D. B. Johnson and D. A. Maltz, "Dynamic source routing in ad hoc wireless networks," Mobile com- puting, pp. 153-181, 1996. 
[5] C. E. Perkins and P. Bhagwat, "Highly dynamic destination-sequenced distance-vector routing (dsdv) for mobile computers," ACM SIGCOMM computer communication review, vol. 24, no. 4, pp. 234-244, 1994, doi: $10.1145 / 190314.190336$.

[6] Z. Haas, "The zone routing protocol (zrp) for ad hoc networks," IETF Internet draft, draft-ietf-manet- zone-zrp-01. txt, 1998.

[7] V. Park, "Temporally-ordered routing algorithm (tora) version 1 functional specification," Internet Draft, draft-ietfmanet-tora-spec-04. txt, 2001.

[8] T. K. Vu and S. Kwon, "Mobility-assisted on-demand routing algorithm for manets in the presence of location errors," The Scientific World Journal, vol. 2014, 2014, doi: 10.1155/2014/790103.

[9] H. Faouzi, H. Mouncif, and M. Lamsaadi, "Aodv energy routing mechanism for multi-channel multi-interface ad hoc networks (emcmi-aodv) using a dynamic programming algorithm," International Journal of Mobile Computing and Multimedia Communications (IJMCMC), vol. 7, no. 4, pp. 1-16, 2016, doi: 10.4018/IJMCMC.2016100101.

[10] R. A. Calvo and J. P. Campo, "Adding multiple interface support in ns-2," University of Cantabria, 2007.

[11] I. L. Cherif, L. Zitoune, and V. Vèque, "Throughput and energy consumption evaluation in directional antennas mesh networks," 2016 IEEE 12th International Conference on Wireless and Mobile Computing, 2016, pp. 1-8, doi: 10.1109/WiMOB.2016.7763211.

[12] N. Ket and S. Hippargi, "Modified aodv energy aware routing for optimized performance in mobile ad-hoc networks," 2016 International Conference on Wireless Communications, Signal Processing and Networking (WiSPNET), 2016, pp. 1030-1034, doi: 10.1109/WiSPNET.2016.7566293.

[13] H. Ashwini, V. R. KP, and I. Ginimav, "Cm-aodv: an efficient usage of network bandwidth in aodv protocol," 2018 International Conference on Design Innovations for 3Cs Compute Communicate Control (ICDI3C), 2018, pp. 111-114, doi: 10.1109/ICDI3C.2018.00032.

[14] A. Abu-Ein and J. Nader, "An enhanced aodv routing protocol for manets," International Journal of Computer Science Issues (IJCSI), vol. 11, no. 1, pp. 54, 2014.

[15] Z. Zhaoxiao, P. Tingrui, and Z. Wenli, "Modified energy-aware aodv routing for ad hoc networks," 2009 WRI Global Congress on Intelligent Systems, vol. 3, pp. 338-342, 2009.

[16] A. Sharma, A. Bansal, and V. Rishiwal, "Sbadr: stable and bandwidth aware dynamic routing protocol for mobile ad hoc network," International Journal of Pervasive Computing and Communications, 2020.

[17] A. Pal, P. Dutta, A. Chakrabarti, J. P. Singh, and S. Sadhu, "Biogeographic-based temporal prediction of link stability in mobile ad hoc networks," Wireless Personal Communications, vol. 104, no. 1, pp. 217-233, 2019.

[18] A. M. Bamhdi, "Efficient dynamic-power aodv routing protocol based on node density," Computer Standards and Interfaces, vol. 70, pp. 103406, 2020. [Online]. Available: http://www.sciencedirect.com/science/article/pii/S0920548919304453.

[19] K. A. Darabkh, M. G. Alfawares, and S. Althunibat, "Mdrma: Multi-data rate mobility-aware aodv-based protocol for flying ad-hoc networks," Vehicular Communications, vol. 18, pp. 100163, 2019, doi: 10.1016/j.vehcom.2019.100163.

[20] H. Faouzi, M. Er-rouidi, H. Moudni, H. Mouncif, and M. Lamsaadi, "Improving network lifetime of ad-hoc network using energy AODV (e-aodv) routing protocol in real radio environments," International Conference on Networked Systems, 2017, pp. 27-39.

[21] A. K. Dogra, et al., "Q-AODV: A flood control ad-hoc on demand distance vector routing protocol," 2018 first international conference on secure cyber computing and communication (ICSCCC), 2018, pp. 294-299, doi: 10.1109/ICSCCC.2018.8703220.

[22] P. Rani and G. Biswas, "AODV enhancement based on the minimization of route-request packets," International Conference on Computer Science and Information Technology, 2012, pp. 442-454.

[23] S. R. Malwe, N. Taneja, and G. Biswas, "Enhancement of DSR and AODV protocols using link availability prediction," Wireless Personal Communications, vol. 97, no. 3, pp. 4451-4466, 2017, doi: 10.1109/ICSCCC.2018.8703220.

[24] S. Ahn, "Aodv extensions for multipath routing, ietf internet draft," University of Seoul, Nov. 2017.

[25] H. Jhajj, R. Datla, and N. Wang, "Design and implementation of an efficient multipath AODV routing algorithm for manets," 2019 IEEE 9th Annual Computing and Communication Workshop and Conference (CCWC), 2019, pp. 0527-0531, doi: 10.1109/CCWC.2019.8666607.

[26] Y. Mai, F. M. Rodriguez, and N. Wang, "Cc-adov: An effective multiple paths congestion control AODV," 2018 IEEE 8th Annual Computing and Communication Workshop and Conference (CCWC), 2018, pp. 1000-1004, doi: 10.1109/CCWC.2018.8301758.

[27] P. Kyasanur and N. H. Vaidya, "Routing and interface assignment in multi-channel multi-interface wireless networks," IEEE Wireless Communications and Networking Conference, 2005, pp. 2051-2056, doi: 10.1109/WCNC.2005.1424834.

[28] W. Su, S.-J. Lee, and M. Gerla, "Mobility prediction and routing in ad hoc wireless networks," International journal of network management, vol. 11, no. 1, pp. 3-30, Feb. 2001, doi: 10.1002/nem.386, doi: 10.1002/nem.386.

[29] E. Hyytiä, H. Koskinen, P. Lassila, A. Penttinen, J. Roszik, and J. Virtamo, "Random waypoint model in wireless networks," Networks and algorithms: Complexity in physics and computer science, vol. 590, Jan. 2005. 


\section{BIOGRAPHIES OF AUTHORS}

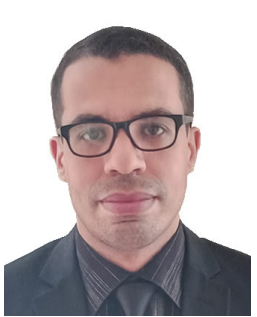

Hassan Faouzi received his undergraduate degrees as well as his bachelor from Hassan $1^{\text {er }}$ University (2011), master's degree (2007), M. Sc. degree in Business Intelligence in 2013 and his doctorate degree in computer science (2018) from Sultan Moulay Slimane University.He has teaching experience of more than 6 years in the areas of networking, development, data mining, etc. His researches are in fields of Ad hoc network, security, machine/deep learning, image processing, and big data. In addition to his scientific career, Dr Hassan participates in the Organizing and scientific committees of the International Conference on Optimization and Applications.

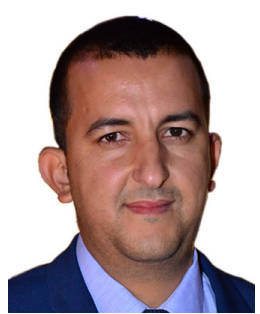

Mohammed Boutalline is currently an Assistant Professor in the National School of Applied Sciences of Beni Mellal at the Sultan Moulay Slimane University in Morocco. Dr Boutalline received his undergraduate degrees as well as his bachelor's degree from Sidi Mohamed Ben Abdellah University in 2009 and his master's and doctorate degrees in computer science from Sultan Moulay Slimane University (2009-2016). His research activities are currently focused on fields: computer science, big data, machine/deep learning. In addition to his scientific career, Dr Mohammed participates in the organization of several conferences namely International Conference on Optimization and Applications (ICOA), International Conference on Business Intelligence (CBI), etc. 